\title{
The effects of different inflation risk premiums on interest rate spreads
}

\author{
Hakan Berument*, Zubeyir Kilinc, Umit Ozlale \\ Department of Economics, Bilkent University, Bilkent, Ankara 06800, Turkey
}

Received 9 April 2003

\begin{abstract}
This paper analyzes how the different types of inflation uncertainty affect a set of interest rate spreads for the UK. Three types of inflation uncertainty-structural uncertainty, impulse uncertainty, and steady-state inflation uncertainty — are defined and derived by using a time-varying parameter model with a GARCH specification. It is found that both the structural and steady-state inflation uncertainties increase interest rate spreads, while the empirical evidence for the impulse uncertainty is not conclusive.
\end{abstract}

(C) 2003 Elsevier B.V. All rights reserved.

PACS: E43; E31; C22

Keywords: Interest rates; Inflation uncertainty; GARCH; Kalman filter

\section{Introduction}

Analyzing interest rate spreads has always been popular among economists. While some academicians use spreads as an indicator of future economic performances (see, Bernanke [1], Stock and Watson [2], Friedman and Kuttner [3-5]), others try to explain the behavior of spreads themselves (see, Chapter 11 of Campbell et al. [6] and the references cited therein) often by testing the expectations hypothesis of the term structure of interest rates.

Although there are some empirical findings that are agreed upon, some studies find conflicting results about the dynamics of the term structure of interest rates (see, Campbell et al. [6] and Christiano et al. [7]). Fuhrer [8] and Chen [9] argue that

\footnotetext{
${ }^{*}$ Corresponding author. Tel.: +90-312-290-2342; fax: +90-312-266-5140.

E-mail address: berument@bilkent.edu.tr (H. Berument).
} 
the reason behind these mixed results stems from the fact that short-term interest rates are not volatile enough to explain long-term interest rates. Moreover, Balduzzi et al. [10] argue that longer-term rates are more heavily influenced by the persistent expectation for future target changes in short-term interest rates, possibly due to expected changes in monetary policy. Thus, the nature of the spreads or their predictive powers for the future economic performance might be influenced by different factors, which concern monetary policy makers. McCallum [11] and Walsh [12] discuss the effect of an exogenous rise in the risk premium on the interest rates, and Evans [13] and Chen [9] report that there is a time-varying inflation risk premium throughout the term structure of interest rates. Thus, uncertainty stemming from inflation is a well-recognized variable in the literature to explain the behavior of interest rates.

Some of the studies mentioned above suggest that inflation uncertainty is an indicator of interest rate spreads. One common factor in these studies is that they stop short of (1) identifying different sources of inflation uncertainty, and (2) observing the effects of these inflation uncertainties on interest rate spreads. ${ }^{1}$ Evans [15] and Berument et al. [16] elaborate three types of inflation uncertainty: structural uncertainty, which arises from the instability of the relationship between current and lag values of inflation; impulse uncertainty, which arises from temporary shocks that hit the economy; and steady-state inflation uncertainty, which arises from the uncertainty on the level of long-run inflation. They show that the effects of these inflation uncertainties on inflation and interest rates can be different.

This study takes the above discussion as its starting point and analyzes the effects of different types of inflation uncertainty on interest rate spreads for the UK. The main reason for choosing UK is the availability of the vast literature devoted to inflation risk in the UK, pioneered by Engle [17]. In order to assess the different types of inflation uncertainty, a time-varying parameter model with a generalized autoregressive conditional heteroskedasticity (GARCH) specification is employed. Such a model allows us to identify different types of inflation uncertainty and observe their effects on interest rate spreads. Section 2 introduces the system of equations that is to be used in modeling the inflation uncertainty. Section 3 reports the estimates. Section 4 of the paper concludes that while the structural uncertainty and the steady-state uncertainty increase the interest rate spreads, the evidence on the effect of the impulse uncertainty on the interest rate spreads is not conclusive. These findings suggest that investors demand higher compensation to hold longer-term and less liquid bonds as the steady-state and the structural inflation uncertainty increase. On the other hand, the inflation uncertainty, which is caused by unexpected temporary shocks to inflation, does not have a uniform effect on the interest rate spreads.

\footnotetext{
${ }^{1}$ To the best of our knowledge, Balaban [14] is the only study that decomposes inflation volatility; he decomposes the inflation volatility by considering its sub-indexes. However, this study is short of assessing the effect of sub-index volatility on the real economic performance.
} 


\section{Modeling inflation uncertainty}

One obvious method for measuring inflation uncertainty is the survey-based approach as employed by Hafer [18], and Davis and Kanogo [19]. This approach measures the uncertainty by taking the standard deviation of inflation forecasts. On the other hand, Bomberger [20] claims that using the dispersion of the survey forecast does not provide a measure of uncertainty; rather this method provides a measure of disagreement. Moreover, forecasters may try to avoid deviating from others' forecasts; this avoidance causes the value of expected inflation to be biased.

A better method could be to employ the Kalman filter approach, which is used to measure the uncertainty about the structural variability in the parameter estimates of an equation. In other words, this approach is capable of measuring inflation uncertainty by estimating the time-varying conditional variance of a variable's parameter estimates.

Finally, one may use a class of autoregressive conditional heteroskedastic models to measure inflation uncertainty. This method allows us to measure the inflation uncertainty by using the conditional variance of the residuals of an inflation specification.

In this study, we combine the last two methods to measure inflation uncertainty by using a time-varying parameter model with a GARCH specification. As it will be clear, such a methodology allows us to identify different types of inflation uncertainty.

Formally, the inflation uncertainty can be modeled as

$$
\begin{aligned}
& \pi_{t+1}=X_{t} \beta_{t+1}+\varepsilon_{t+1}, \quad \text { where } \varepsilon_{t+1} \sim N\left(0, h_{t}\right), \\
& \beta_{t+1}=\beta_{t}+v_{t+1}, \quad \text { where } v_{t+1} \sim N(0, Q), \\
& h_{t}=\bar{h}+\sum_{i=0}^{m} \phi_{i} \varepsilon_{t-i}^{2}+\sum_{i=1}^{n} \gamma_{i} h_{t-i},
\end{aligned}
$$

where $\pi_{t}$ is the inflation rate; $X_{t}$ is the $l \times 1$ vector for a constant term and $l-1$ explanatory variables for inflation; $\varepsilon_{t}$ is the normally distributed error term with a time-varying conditional variance of $h_{t}$ at time $t$. Here, Eq. (1) is for the inflation process, Eq. (2) is for the development of the estimated parameters of the inflation equation, and Eq. (3) is for the conditional variance of the inflation equation residuals. In particular, in the above model, $\beta_{t+1}$ is the $l \times 1$ parameter vector of the inflation specification; $v_{t+1}$ denotes the vector of shocks to $\beta_{t+1}$; and $v_{t+1}$ has a normal distribution with a homoskedastic covariance matrix of $Q$. After constructing the model, we now include the Kalman filter updating equations in the above specification in order to assess the uncertainty measures. Particularly, we modeled the inflation process as

$$
\begin{aligned}
& \pi_{t+1}=X_{t} E_{t} \beta_{t+1}+\eta_{t+1}, \\
& H_{t}=X_{t} \Omega_{t+1 \mid t} X_{t}^{\prime}+h_{t}, \\
& E_{t+1} \beta_{t+2}=E_{t} \beta_{t+1}+\left[\Omega_{t+1 \mid t} X_{t}^{\prime} H_{t}^{-1}\right] \eta_{t+1}, \\
& \Omega_{t+2 \mid t+1}=\left[I-\Omega_{t+1 \mid t} X_{t}^{\prime} H_{t}^{-1} X_{t}\right] \Omega_{t+1 \mid t}+Q .
\end{aligned}
$$


The conditional covariance matrix of $\beta_{t+1}$, which represents the structural uncertainty of the inflation process, is denoted by $\Omega_{t+1 \mid t}$. Then, Eq. (5) accounts for the two types of uncertainty, which originate from inflation shocks $\left(\varepsilon_{t+1}\right)$ and the structure of the inflation $\left(v_{t+1}\right)$. Eq. (4) is used for forecasting the future inflation and Eq. (6) shows the innovations in updating the estimates of $\beta_{t+2}$. Also, Eq. (7) denotes the conditional distribution of $\beta_{t+2}$ and give us the new parameter vector based on new information.

In the model presented above, $\varepsilon_{t+1}$ can be viewed as describing the shocks that hit the economy. Then, the time-varying parameter $\beta$ will show how these shocks are propagated through the economy. Such a terminology leads us to Frisch and Slutsky's distinction between impulses and propagation (for a detailed discussion, see Blanchard and Fisher [21, p. 277]). Then, we can refer to the uncertainty associated with the randomness in $\beta$ as the structural uncertainty, which we measured by $X_{t} \Omega_{t+1 \mid t} X_{t}^{\prime}$, while the uncertainty associated with randomness in $\varepsilon_{t+1}$ becomes the impulse uncertainty, which is measured by the conditional variance of $\varepsilon_{t+1}\left(h_{t}\right)$.

\subsection{Justification of the model}

The purpose of this sub-section is to justify the selection of the GARCH-Kalman filter specification used in this paper. Following Berument [22] and Grier and Perry [23], we model the inflation variable as an autoregressive (AR) process. The lag order is selected by the final prediction error criteria (FPE). The FPE selects the lag order such that the residuals of the inflation equation are no longer autocorrelated. This is important because ARCH-LM tests of autocorrelated residuals wrongly suggest the presence of an ARCH effect even if there is no such effect (see, Jansen and Cosimona, [24]). The FPE criteria suggest that the lag order of the UK inflation variable should be two. Next, we estimate the inflation equation as an $\operatorname{AR}(2)$ process and apply the ARCH-LM tests for various lag orders. The ARCH-LM test statistics for 1, 6, and 12 lags are 29.76, 50.05, and 58.01, respectively. These results clearly suggest the presence of the ARCH effect. Various specifications of GARCH are considered next. GARCH $(1,1)$ is selected as the process to assess the conditional variance of the inflation equation.

Next, besides the random walk specification (Eq. (2)), two other time-varying parameter specifications are also estimated for the inflation equation parameters: returnto-normality and constant mean. The return-to-normality in parameter specification can be written as

$$
\left(\beta_{t+1}-\bar{\beta}\right)=F\left(\beta_{t}-\bar{\beta}\right)+v_{t+1}
$$

and the constant mean specification can be written as

$$
\beta_{t+1}=F \bar{\beta}+v_{t+1} .
$$

These three specifications are estimated; however, Table 1 suggests that the random walk specification used in this study outperforms its alternatives, in terms of both Schwarz information criteria (SIC) and Akaike information criteria (AIC). 
Table 1

Model selection criteria

\begin{tabular}{llr}
\hline Models & AIC & SIC \\
\hline Random walk & 3.76 & 3.88 \\
Return-to-normality & 4.03 & 4.14 \\
Constant mean & 4.68 & 4.73 \\
\hline
\end{tabular}

\subsection{The steady-state inflation uncertainty}

Besides the structural and impulse uncertainties, the steady-state inflation uncertainty is also introduced as the third type of uncertainty. When the inflation equation is specified as

$$
\pi_{t+1}=\beta_{1, t+1}+\beta_{2, t+1} \pi_{t}+\beta_{3, t+1} \pi_{t-1}+\varepsilon_{t+1},
$$

the steady-state inflation can be defined as

$$
\pi_{t+1}^{*}=\left(1-\beta_{2, t+1}-\beta_{3, t+1}\right)^{-1} \beta_{1, t+1} .
$$

Therefore, the conditional variance of the steady-state inflation is

$$
\nabla_{t}^{2}\left(\pi_{t+1}^{*}\right)=\nabla\left(E_{t} \beta_{t+1}\right) \Omega_{t+1} \nabla\left(E_{t} \beta_{t+1}\right)^{\prime}
$$

where

$$
\nabla\left(E_{t} \beta_{t+1}\right)^{\prime}=\left[\begin{array}{c}
{\left[\left(1-E_{t} \beta_{2, t+1}-E_{t} \beta_{3, t+1}\right)\right]^{-1}} \\
E_{t} \beta_{1, t+1}\left[\left(1-E_{t} \beta_{2, t+1}-E_{t} \beta_{3, t+1}\right)\right]^{-2} \\
E_{t} \beta_{1, t+1}\left[\left(1-E_{t} \beta_{2, t+1}-E_{t} \beta_{3, t+1}\right)\right]^{-2}
\end{array}\right] .
$$

\section{Estimation results}

\subsection{Data set and the interest rate spreads}

After defining the three types of uncertainty measures, we now introduce the data employed in this study. We use the monthly UK data from 1961:06 to 2002:02. Inflation is defined as the logarithmic first difference of the seasonally adjusted CPI. Several interest rates - which vary in terms of liquidity, tax and their responsiveness to market conditions - are used to calculate various interest rate spreads. These interest rates are the overnight interbank minimum interest rate $(R f)$, the Treasury bill rate $($ Rter $)$, the Treasury bill rate bond equivalent (Rterbond), the deposit rate (Depo), the lending rate of clearing banks (Lending), the short-term government bond yields (Gbond), and the long-term government bond yields (Gbondlong). The interest rate spreads are calculated by subtracting the overnight interbank minimum interest rate (which has the shortest maturity) from the remaining six interest rates. 
Table 2

Regression results for the interest rate spreads

\begin{tabular}{lrrrr}
\hline & Constant & \multicolumn{1}{l}{$h_{t}$} & $X_{t} \Omega_{t+1 \mid t} X_{t}^{\prime}$ & $\nabla_{t}^{2}\left(\pi_{t+1}^{*}\right)$ \\
\hline Rter-Rf & 0.009 & 0.009 & 0.123 & 0.061 \\
& $(0.29)$ & $(0.16)$ & $(2.37)$ & $(2.42)$ \\
Rterbond—Rf & -0.019 & 0.010 & 0.132 & 0.078 \\
& $(-0.92)$ & $(0.19)$ & $(2.54)$ & $(3.12)$ \\
Depo-Rf & -0.088 & -0.023 & 0.077 & 0.045 \\
& $(-2.49)$ & $(-0.80)$ & $(2.34)$ & $(2.07)$ \\
Lending—Rf & 0.043 & -0.012 & 0.091 & 0.056 \\
& $(1.89)$ & $(-0.36)$ & $(2.62)$ & $(2.95)$ \\
Gbond_Rf & 0.060 & 0.018 & 0.170 & $(1.66)$ \\
& $(1.38)$ & $(0.23)$ & $(2.41)$ & 0.094 \\
Gbondlong—Rf & 0.040 & 0.050 & 0.238 & $(1.99)$ \\
& $(0.74)$ & $(0.45)$ & $(2.38)$ & \\
\hline
\end{tabular}

\subsection{Empirical evidence}

Finally, after assessing the three inflation uncertainty measures, we can observe the correlation between the six interest rate spreads and these uncertainties. For this purpose, we estimate the following equation:

$$
\text { Spread }_{t}=\lambda_{0}+\lambda_{1} h_{t}+\lambda_{2} X_{t} \Omega_{t+1 \mid t} X_{t}^{\prime}+\lambda_{3} \nabla_{t}^{2}\left(\pi_{t+1}^{*}\right)+z_{t} .
$$

It should be noted that $h_{t}, X_{t} \Omega_{t+1 \mid t} X_{t}^{\prime}, \nabla_{t}^{2}\left(\pi_{t}^{*}\right)$, and $z_{t}$ denote the impulse uncertainty, the structural uncertainty, the steady-state inflation uncertainty and the i.i.d error term, respectively. The estimates are reported in Table 2. Note that where their standard errors are calculated by using the Newey-West's heteroskedastic consistent formula, the $t$-statistics are reported in parenthesis under the corresponding coefficients. We estimate the inflation equation and the three uncertainty measures jointly by using the rolling-regression method. Then, we include these uncertainty measures to estimate Eq. (11). The fact that we do not estimate Eq. (11) along with other equations is due to the unavailability of the full-sample observations for the mid-sample periods. In particular, if we must estimate Eq. (11) along with the uncertainty measures, we need to estimate all the equations at once. However, doing this would suggest that agents knew all the observations for the full sample to obtain the mid-point estimates for the three uncertainty variables; however, this is not true.

Table 2 suggests that the estimated coefficients of the structural and steady-state uncertainties are always positive and statistically significant; however, the estimated coefficients of the impulse uncertainty variable have mixed signs and are not statistically significant. $^{2}$ Therefore, increases in the structural uncertainty and the steady-state uncertainty increase spreads. This supports the proposition that risk-averse investors want

\footnotetext{
${ }^{2}$ The level of significance is at the $5 \%$ level, unless otherwise noted.
} 
to be compensated for bearing higher risk. While the highest compensation requested by investors is on the spread between the long-term government bond yields and the interbank rate, the lowest compensation is on the spread between the deposit and the interbank rate for both the structural and steady-state inflation uncertainties. Moreover, a similar pattern on the order of the spread variables from highest to lowest compensation requested by investors is observed for the effects of these two uncertainties on the six spreads. Thus, we can conclude that these two uncertainties affect the different risk premiums similarly, which ultimately dictate the spreads.

On the other hand, even if the estimated coefficients of the impulse uncertainty are not statistically significant, the evidence is mixed. The impulse uncertainty decreases the deposit-interbank rate spread and the lending-interbank rate spread. On the contrary, the impulse uncertainty increases the other four spreads. Thus, it leads us to conclude that the impulse uncertainty of inflation does not affect the interest rate spreads similarly.

\section{Conclusion}

There is an extensive literature that studies relationships between interest rate spreads and various macroeconomic variables. Within this context, some of these works analyze the predictive power of interest rate spreads for future economic performance, while some others attempt to explain the dynamics of the term structure itself. Moreover, some argue that inflation uncertainty is one of the variables that explains the behavior of interest rate spreads. However, different types of inflation uncertainty may affect the interest rate spreads differently.

This study investigates the effects of different types of inflation uncertainty on various interest rate spreads. The time-varying parameter model with a GARCH specification is employed to derive three different types of uncertainties. These are the structural uncertainty, which indicates uncertainty about the structure of inflation process; the impulse uncertainty, which arises due to the nature and magnitude of the temporary shocks that hit the economy; and the steady-state inflation uncertainty, which is the uncertainty about the level of long-run inflation that ultimately determines the long-run real returns.

This paper argues that the structural uncertainty and the steady-state uncertainty increase the spreads between the six interest rates and the overnight interbank minimum interest rate. Therefore, we can conclude that investors demand higher returns due to increasing levels of the structural and steady-state inflation uncertainties. On the other hand, the evidence regarding the effect of the impulse uncertainty on the interest rate spreads is not conclusive.

\section{Acknowledgements}

We would like to thank Michael Claxton, Martin Evans and Peter N. Ireland for their invaluable suggestions. 


\section{References}

[1] B.S. Bernanke, On the predictive power of interest rates and interest rate spreads, New England Economic Review, Federal Reserve Bank of Boston, 1990, pp. 51-68.

[2] J.H. Stock, M.W. Watson, Interpreting the evidence on money-income causality, J. Econometrics 40 (1) (1989) 161-181.

[3] B.M. Friedman, K.N. Kuttner, Money, income, prices and interest rates, Amer. Econ. Rev. 82 (3) (1992) 472-492.

[4] B.M. Friedman, K.N. Kuttner, Economic activity and the short-term credit markets: an analysis of prices and quantities, Brookings Pap. Econ. Activity 0 (2) (1993) 193-266.

[5] B.M. Friedman, K.N. Kuttner, Indicator properties of the paper-bill spread: lessons from recent experience, Rev. Econ. Statist. 80 (1) (1998) 34-44.

[6] J.Y. Campbell, A. Lo, A. MacKinlay, Econometrics of Financial Markets, Princeton University Press, Princeton NJ, 1997.

[7] L. Christiano, M. Eichenbaum, C.L. Evans, Monetary policy shocks: what have we learned and to what end?, in: J.B. Taylor, M. Woodford (Eds.), Handbook of Macroeconomics, Vol. 1A, Elsevier, Amsterdam, Netherlands, 1999.

[8] J.C. Fuhrer, Monetary policy shifts and long-term interest rates, Quart. J. Econ. 111 (4) (1996) 1183-1209.

[9] L. Chen, Inflation and real short-term interest rates - a Kalman filter analysis of the term structure, Appl. Econ. 33 (2001) 855-861.

[10] P. Balduzzi, G. Bertola, S. Foresi, L. Klapper, Interest rate targeting and the dynamics of short-term rates. NBER Working Paper 5944, February, 1997.

[11] B.T. McCallum, Monetary policy and the term structure of interest rates, NBER Working Paper, No. 4938, November, 1994.

[12] C.E. Walsh, Monetary Theory and Policy, MIT Press, Cambridge MA, 1998.

[13] M. Evans, Real rates, expected inflation, and inflation risk premia, J. Finance LIIL (1) (1998) 187-218.

[14] E. Balaban, Core inflation measurement: a new approach, Mimeo, 1999.

[15] M. Evans, Discovering the link between inflation rates and inflation uncertainty, J. Money Credit Banking 23 (1991) 169-184.

[16] H. Berument, Z. Kilinc, U. Ozlale, The missing link between inflation uncertainty and interest rates, Mimeo, 2002.

[17] R. Engle, Autoregressive conditional heteroscedasticity with estimates of the variance of United Kingdom inflation, Econometrica 50 (1982) 987-1007.

[18] R.W. Hafer, Inflation uncertainty and a test of the Friedman Hypothesis, J. Macroecon. 8 (3) (1986) $365-372$.

[19] G. Davis, B. Kanogo, On measuring the effect of inflation uncertainty on real GNP growth, Oxford Econ. Pap. 48 (1996) 163-175.

[20] T. Bomberger, Disagreement as a measure of uncertainty, J. Money Credit Banking 28 (3) (1996) 381-392.

[21] O.J. Blanchard, S. Fischer, Lectures on Macroeconomics, MIT Press, Cambridge MA, 1989.

[22] H. Berument, The impact of inflation uncertainty on interest rates in the UK, Scott. J. Political Econ. 46 (1999) 207-218.

[23] K.B. Grier, M.J. Perry, The effects of real and nominal uncertainty on inflation and output growth: some GARCH-M evidence, J. Appl. Econometrics 15 (2000) 45-58.

[24] D.W. Jansen, T.F. Cosimona, Estimates of the variance of US, Inflation based upon the ARCH model, J. Money Credit Banking 20 (3) (Part 1) (1988) 409-421. 